\title{
A GUIDELINE For AN EFFECTIVE USER INTERFACE FOR EDUCATIONAL SEMANTIC APPLiCATION
}

\author{
Tinashini Jayadevan Naidu and Aslina Saad \\ Computing Department, Faculty of Art, Computing \& Creative Industry, \\ Universiti Pendidikan Sultan Idris, 35900, Tanjong Malim, Perak, Malaysia
}

\begin{abstract}
This research aims to produce a guideline for an effective user interface for semantic application particularly educational. In order to develop the guideline, a methodology of four phases; analysis, design, development and evaluation has been applied. Qualitative approach was used by using checklist instrument and interview questions with the experts in system design. A prototype was then developed using the proposed guideline. The prototype was evaluated using qualitative approach based on heuristic evaluation instrument involving a sample of ten teachers of secondary level. The user acceptance for this system was compared to the one which has been developed without the guideline. The result shows that the system developed by the proposed guideline has higher acceptance. Seven of ten teachers agreed that the most important principle in user interface design is effectiveness specifically the simplicity, aesthetic and minimalist design. It is useful to develop an effective user interface using this guideline.
\end{abstract}

\section{KEYWORDS}

Interface, Education, Semantic application, User interface guideline, Educational Semantic Web

\section{INTRODUCTION}

Educational systems around the world have been experiencing changes and reforms. Stepping into the new millennium, many societies around the world are engaging in serious and promising educational reforms. Similar tailoring based on learners' interests; strengths and needs could be applied in teaching and learning environments. Semantic Web tools can give each teacher candidate or student the ability to process information with their own pace [4]. The latest effort used to overcome all the constraints is a web-based system which has semantic features for customising lesson plans. Main contribution in terms of education semantic web can give is its advanced search capability. This because the Semantic Web allows "its users to find relationships between tagged information using inference rules and data organizational tools called ontologies that provide logic and structure to the information embedded in web pages" [14].

Referring to the phases of semantic content in the educational application, the researcher found that there have been various progresses on the backend part in terms of data storage and data linking. Nevertheless, the least concerning part and developed in the educational semantic web application is the front end which is the user interface. It is found that less effort are given in designing the interface part of the system compared to the other back end work of construction in the application. There are massive works 
The International Journal of Multimedia \& Its Applications (IJMA) Vol.10, No.6, December 2018 that have been carried out in the backend of the system in term of fulfilling the functionalities and the system requirements yet, there is no improvement for the front space, which is the interface.

Due to the effectiveness of the applications that supports pedagogical research is given more attention as their capability to support IT tools compared to the manual data retrieving methods. The complexity of real life learning situation are gaining back its opportunity as the using technology in pedagogical research trend is enhancing. Thus, in conjunction to this situation it is in need of a system with rich synchronized user friendly interaction required [15]. The user interface serves as an essential part of the program because it serves as the medium that connects program to users. Even with having the best application function, if it still with a bad user interface, then the end result will still be bad. The interface acts a key point to evaluate whether the application is being accepted in market [16]. It was proven in a study that 48 percent of the source code is conquered by the user interface. As more and more applications and functions are related to real world, the importance of a good user interface is still undeniable [3]. A research was carried out on an existing semantic web.

The purpose of this study is to investigate the effectiveness of the user interface of educational semantic web application, with the following objectives:

- To produce a user interface guideline that supports educational semantic web application

- To design an interface using the produced user interface guideline that supports educational semantic web

- To evaluate the user acceptance towards the developed prototype based on produced user interface guideline

To enlighten the readers in order to follow through the development process of this research, this paper is organized as follows: Section 2 discusses the review of literature related to evolutions of web, educational system, interface design and the previous application (iRPH), Section 3 elaborates the four phases of research methodology in the study, Section 4 discusses the data analysis and findings, and Section $5 \& 6$ concludes and summarizes the discussion of the study.

\section{Literature REVIEW}

The diffusion of web technologies has led to significant changes in the educational field and the quality of learning. Web 1.0 allowed the development of web-based systems, characterised by read-only educational resources. Later, Web 2.0 made possible the creation of interactive and collaborative educational systems where users can participate in synchronous and asynchronous communications and real-time group learning activities through wikis, blogs, social networking tools and online virtual environments [8]. However, the usefulness of any technology in any field is dependent on its capacity to address real problems and practical needs in that field [7]. Finding shows, Web 2.0 fails to offer an adaptive and personalized web-based learning environment and address individual learning needs. Personalization manages to customize the instruction process according to the learner's background knowledge, skills, learning objectives, motivations and preferred activities. On the other hand, adaptation, to some extent, is what makes personalisation possible through the fully or partially automated, software supported collection and analysis of individual learners' characteristics [10] reported that Web 3.0 promises to assist researchers and practitioners alike toward addressing the aforementioned educational needs by adding 
The International Journal of Multimedia \& Its Applications (IJMA) Vol.10, No.6, December 2018

'intelligence' in the field of technology enhanced and web based education and helping computers in understanding the available educational material and there is where the semantic technology is applied. The next paragraph elaborates the four of the applications of semantic web in the field of learning, specifically those of learning objects, learning object repositories and pedagogical agents that are considered as the applications of vital importance toward benefiting the field of education and learning.

A major drawback of the semantic approach is that ontological knowledge is only used to produce a keyword form which is only understandable by the experts and the user is still left to guess what are the keywords and what will result in the instances sought [1]. At a glance it can be clearly seen that there are difficulties at the interface level, whereby the ontology keyword in the semantic web is not being presented to user in an appropriate and understandable way. Semantic web had seen a few breakthrough researches over the recent years. A study had been done intensively on semantic webs in various aspects. The finding shows that less effort are given in developing the interfaces compared to the other types of construction in the system [9]. There are massive work have been carried out in the backend of the system in term of making the functionalities and the system needs yet, there is no provision for the front space the interface as the interface is the major medium where the interaction happens. Important issues that had been pointed out is that the users are looking for in depth functionalities for a particular application, which these semantic web with the function that is also known as 'all in one' (unified) interfaces are not capable of providing [9]. In extending this issue, to enable a user friendly user interface each function should be provided with each effective interface. The lacking point in educational semantic web interfaces is none other than motivation, whereby motivation is what causes us to act [28]. Pedagogic stimulus should not involve fear of punishment or desire for reward, but it should involve willingness that will trigger the deep desire to learn something. . From these issues it is cleared that very little attention has been focused on these above matters in the quest of creating a user friendly user interface. After encountering various aspects of semantic web it is convinced without any denial that most of the remaining challenges to realize the Semantic Web vision have nothing to do with the underlying technologies. Instead, it all comes down to user interfaces and usability [29]. This clearly shows that the main issues that have been popping out is the issue of usability and interfaces because that is the only medium used by the users to access these websites. User interface is the point of interaction between user and educational body.

The aims of education are not achieved due to unsuccessful correlations, even if the educational content was selected well and the user is willing to learn [6]. The minus point in teaching and learning is because of poor interaction. The findings of the study indicated that the disclosures are mainly concentrated on the user interface. Challenges for the semantic application regard usability [18]. To facilitate the use of the framework, especially for non-technical users, it is necessary to improve the graphical interface and to develop more complex fault tolerance mechanisms in terms of the user interfaces.

When it comes to educational semantic web, the lacking of interaction between the user who is addressed as the educational body and the interface brings to poor interaction which leads to failure in the teaching and learning session. The existing semantic application for lesson planning shows that quite some progress on the backend side in terms of storing structured content or for linking data but least developed aspect of the online lesson planning application is from the user interface aspect [2]. To develop a prototype CBR application for lesson planning i-RPH system, a semantic application that applied the constructed ontology has been developed as a prototype 
The International Journal of Multimedia \& Its Applications (IJMA) Vol.10, No.6, December 2018 system for lesson plan construction.In order to support retrieval of relevant lesson plan [13]the main aim of the application is that it was developed to proof the constructed ontology is applicable. Thus the functional aspect of system development was given priority compared to the non-functional aspect which includes usability mainly the user interface part.

\section{METHODOLOGY}

This research involves three main objectives which is designing the semantic user interface guideline, developing the prototype of the interface by applying the guideline that has been produced. Finally, the evaluation result of the designed user interface will be presented in the next chapter. Figure 1 below illustrates the research methodology phases use throughout this research.

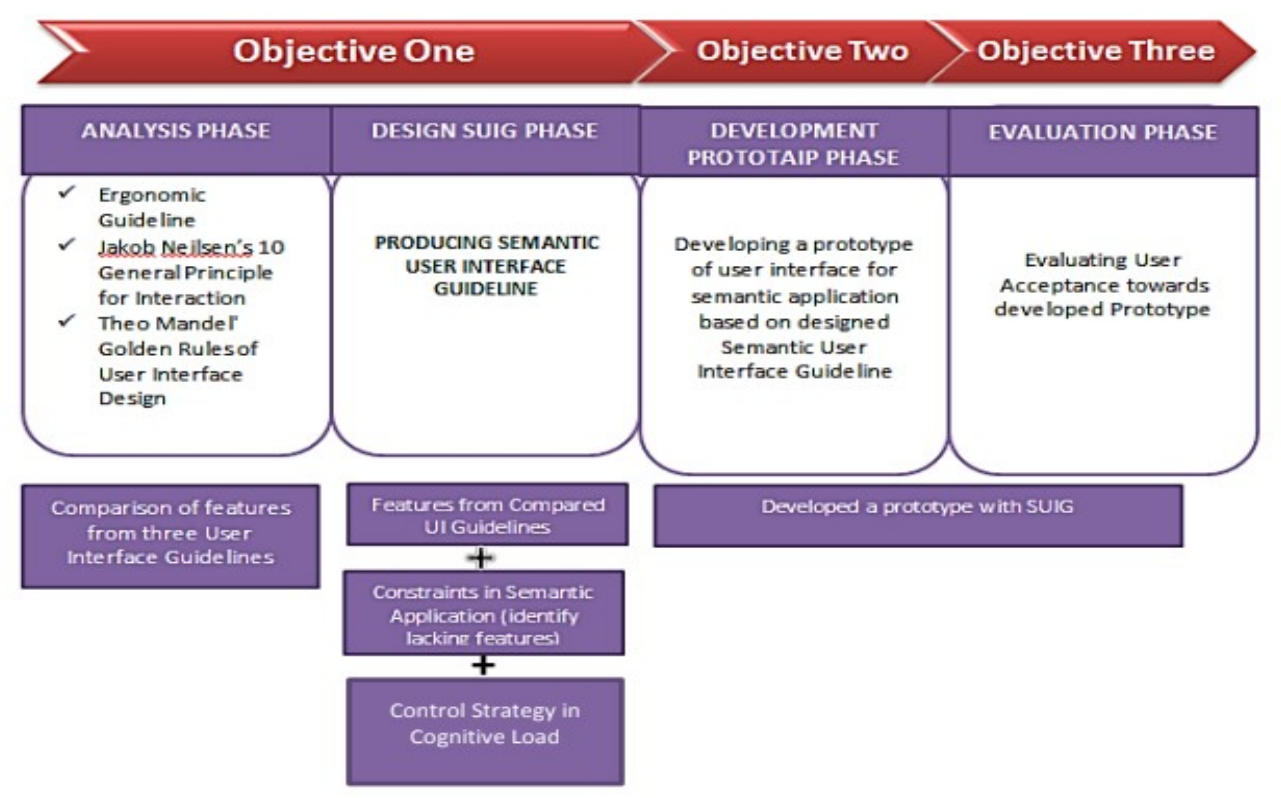

Figure 1. Four phases in the research methodology

\section{Phase 1: AnAlysis}

The first method in the analysis phase is to decide whether the problem can be solved by carrying out needs assessment. The need assessments in this research are investigated via previous researches with surveys and studies on research and example projects done by other researchers or developer. Basically, needs assessment is the method to identify problems and the causes, then finding solutions to those problems (Dick and Carey, 2009). There are two highlighted concepts in this research.

The user interface for semantic application and user interface guideline, which is widely used in educational web. The research gap is also investigated based on the previous projects and research journals derived from the ISI, IEEE, Web of Science and Scorpus. This research gap is then presented in the Research Territory Map (RTM) (refer to chapter 1). From the obtained user interface guidelines, a comparison of three main use interface guideline will be carried out. 
The International Journal of Multimedia \& Its Applications (IJMA) Vol.10, No.6, December 2018

\section{PhASE 2: DESIGN}

The design phase involves the method of embedding the results from the analysis phase to plan a framework for designing a user interface for educational semantic web application. The design phase in ADDIE instructional design obtains the output from the analysis phase and then builds a user interface for educational semantic web application. This phase needs logical and creative thinking as it involves the ability to get into the mind of the users.

\section{SEMANTIC USER INTERFACE GUIDELINE (SUIG)}

Figure 2 shows the process of the designing phase, wherein a comparison of different interface models/concepts is carried out. The models compared include Ergonomic Guideline, Jacob Neilson 10 General Principle of Interaction and Theo Mandel's Golden Rules of User Interface. The common features in all three user interface guidelines are then gathered. Issues regarding user interface of semantic applications are analysed and based on these issues, the features concerned is rectified. A control strategy for cognitive load extracted from cognitive load theory was designed. For content and subject matter, from analysed features from the user interface guideline constraints, as well as the control strategy for cognitive load are embedded to design a user interface guideline for educational semantic application.

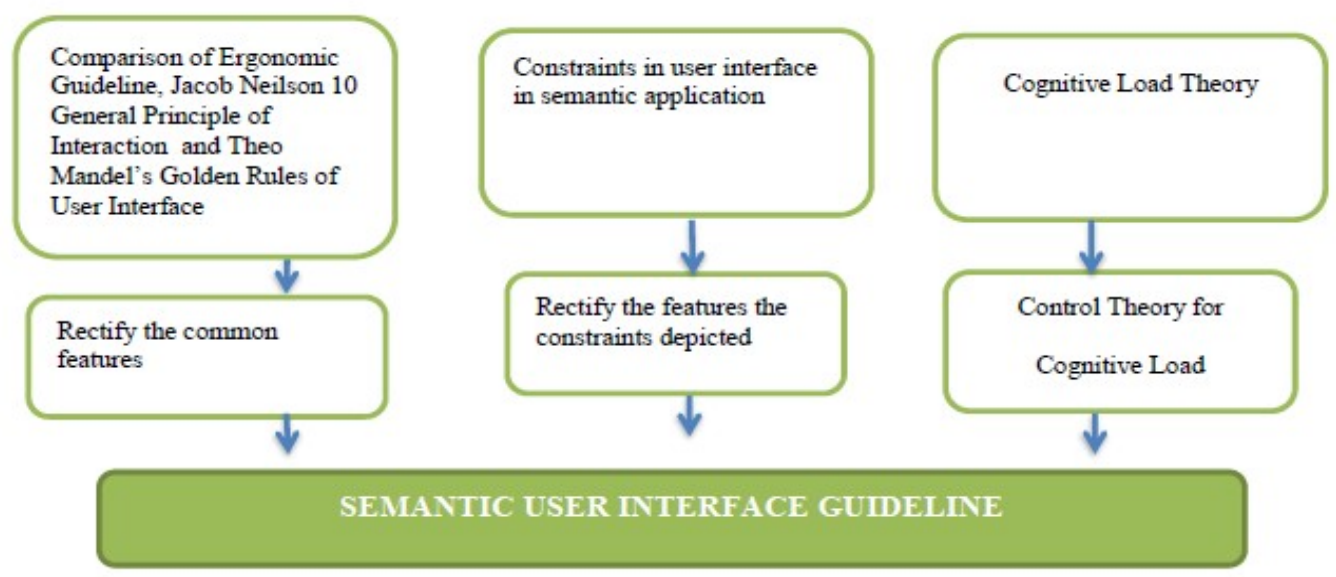

Figure 2. Designing user interface guideline

\section{Phase 3: DeVElopment}

The development phase is where the researcher creates and intergrates the content that was created in design phase. The researcher then works towards the development of integrate technologies. The Development phase includes the following:

\section{Prototype Design}

After all the above details needed are identified, a prototype has been developed. The prototype will be given to the users to be used and tested. The user will be giving comments on the evaluated prototype. From the user's feedback the prototype will be revised and implemented according to user's suggestion. These steps will be repeated until the users are satisfied with the prototype. 
The International Journal of Multimedia \& Its Applications (IJMA) Vol.10, No.6, December 2018

\section{Prototype Development}

During this phase, all the software needed to develop the user interface design for semantic application for lesson planning was identified. As for this user interface design, Adobe Dreamweaver CS6 was used. The web programming's that will be used for development of the user interface are HTML PHP, AJAX, CSS and JavaScript. The developed prototype is then evaluated for user acceptance.

\section{Phase 4: Evaluation}

This section discusses the result from the user acceptance evaluation. In this evaluation phase, users are given opportunity to use two semantic applications. One of the applications is designed without any user interface guideline whereas the other semantic application is designed with Semantic User Interface Guideline.

\section{FINDING}

\subsection{Semantic USER Interface Guideline (SUIG). (OBJECTIVE 1)}

This section depicts the chronology of the process involved in producing the SUIG, Semantic User Interface Guideline. In the previous section, a comparison table has been made to identify elements to be embedded in the guideline. Three user interface guideline is choose to be compared based on the justification below:

Ergonomic Guidelines -To adapt ergonomic elements and their usage to improve user information processing in terms of improved efficiency, fewer errors and accidents, and increased well-being. It is also used to synthesise recommendations for design, and to evaluate uses and recommendations [12].

Jacob Neilson 10 General Principle of Interaction - This guideline uses a set of simple principles or heuristics, can be systematically accessed to identify problems and their severity, as well consequences for the user. The guideline is suitable for educational wed or application.

Theo Mandel's Golden Rules of User Interface- the main objective of this guideline is to build an interface that adapt to user as in to place the user in control of the interface as well as reduce user's memory.

There are three main features depicted in this analysis table which is Usability, Effectiveness and Interactivity. The justification of this derivation is obtained from the constraints found in the previous research. All the constraint of user interface in semantic application are lacking of this three features. To detect the elements that are suitable in semantic user interface guideline, a comparison Table 1 was made.

In order to decrease cognitive load in Semantic User Interface Design, a control strategy was designed. The solution to decrease the cognitive load of a user design is by decreasing Intrinsic

Cognitive Load (ICL), decreasing extraneous cognitive load (ECG) and increase Germane Cognitive Load (GCL). High level of Germane Cognitive Love (GCL) is created by ease of use contributing to the control strategy. 
The International Journal of Multimedia \& Its Applications (IJMA) Vol.10, No.6, December 2018 Table 1. Comparison of Elements from Ergonomic, Jacob Neilson 10 General Principle of Interaction and

Theo Mandel's Golden Rules of User Interface

\begin{tabular}{|c|c|c|c|}
\hline & $\begin{array}{l}\text { Ergonomic } \\
\text { Guidelines }\end{array}$ & $\begin{array}{llr}\text { Jakob Nielsen's } & 10 \\
\text { General Principle for } & \text { for } \\
\text { Interaction } & \end{array}$ & $\begin{array}{l}\text { Theo Mandel Golden } \\
\text { Rules of User Interface } \\
\text { Design }\end{array}$ \\
\hline \multicolumn{4}{|c|}{ Usability } \\
\hline Learnability & 7 & 7 & 7 \\
\hline Flexibility & 7 & 7 & 7 \\
\hline \multicolumn{4}{|l|}{ Robustness } \\
\hline Satisfaction & 7 & 7 & 7 \\
\hline \multicolumn{4}{|c|}{ Effectiveness } \\
\hline $\begin{array}{l}\text { Simplicity - Aestathic - } \\
\text { Minimalist Design }\end{array}$ & l & 7 & 7 \\
\hline Visual appeal & 7 & & \\
\hline Ease of use & 7 & 7 & 7 \\
\hline \multicolumn{4}{|c|}{ Interactivity } \\
\hline Navigation & 7 & 1 & 1 \\
\hline Consistency & 7 & 7 & 7 \\
\hline Cognitive Loads & 7 & 7 & 7 \\
\hline $\begin{array}{l}\text { Error Prevention, } \\
\text { Detection and Recovery }\end{array}$ & 7 & 7 & 7 \\
\hline Affordance & 7 & 7 & 7 \\
\hline Perceivability & 7 & & \\
\hline
\end{tabular}

Table 2. A control strategy

\begin{tabular}{|l|c|}
\hline COGNITIVE LOAD & STRATEGY \\
\hline DECREASING Intrinsic Cognitive Load(ICL) & $\begin{array}{l}\text { Instruction should be clear and precise } \\
\text { Sequence of process available in } \\
\text { application }\end{array}$ \\
& $\circ$ Limited variety of typography \\
\hline DECREASING Extraneous cognitive load & $\circ$ Avoiding redundant information \\
(ECG) & $\circ$ Content should be interpreted clearly \\
& $\circ$ Avoiding redundant link \\
& $\circ$ Avoid long messages or explanation \\
\hline INCREASING Germane Cognitive Load(GCL) & $\circ$ Clear explanation capture \\
& $\circ$ Present material in a way that cap attention \\
& $\circ$ Timely feedback \\
\hline
\end{tabular}

Undeniably, the semantic application aims to upgrade the classical web by projecting directly on the knowledge embedded in it and refine according to web educational systems which results to the contribution of the upgrade of the learning efficiency. In contrary, the constraint in semantic application is the user interface itself.

\section{Problem Statements}

The following are the constraint highlighted in previous studies:

(a) At a glance, it can be clearly seen that there are difficulties at the interface level, whereby the ontology keyword in the semantic web is not being presented to user in an appropriate and understandable way [1].

(b) There is no provision for the front space the interface, in which it should be noted that the interface is the major medium where the interaction happens [9]. 
The International Journal of Multimedia \& Its Applications (IJMA) Vol.10, No.6, December 2018

(c) Semantic web, with the function that is also known as 'all in one' (unified) interfaces, are not capable of providing the needs of the users. Hence, to enable a user-friendly interface, each function should be provided with effective interface [9].

(d) The lacking point in educational semantic web interfaces is none other than motivation [28].

(e) The main issues that have been recurring are the issue of usability and interfaces because that is the only medium used by the users to access these websites [29].

(f) The lack of interaction between the user (or the educational body) and the interface brings about poor interaction, which leads to failure in the teaching and learning session [6].

( $g$ ) It is not often the case that ontology resources are followed by human understandable lexicalisations [20]

(h) Data but least concentrated aspect of the application is the user interface in the existing semantic lesson plan application [2]

(i) A large amount of structured data adheres to the RDF (Research Description Framework) data model being published on the web. However, it is difficult to discover, access and explore this data [21].

(j) The main concern in semantic application is its usability [22].

(k) The user interfaces is usually overlooked in the semantic web application. This area definitely needs sophisticated visualization as well as interaction techniques to help its users able to understand this area [23].

\section{SEMANTIC USER INTERFACE GUIDELINE (SUIG)}

This section depicts the chronology of the process involved in producing the SUIG, Semantic User Interface Guideline. In the previous section, a comparison table has been made to identify elements to be embedded in the guideline and control strategy of cognitive load was made. Based on these identified elements and constraints in semantic applications as well as applying control strategy for cognitive load, a user interface guideline for Semantic application is designed. Figure 3 shows the process in embedding the rectified elements in user interface and constraints found in semantic applications to create a Semantic User Interface Guideline. The common features in all three user interface guidelines from the comparison table in Table 4.1 are gathered. Issues regarding user interface of semantic applications are analysed and based on these issues, the features concerned is rectified. For content and subject matter, from analysed features from both the user interface guideline as well as constraints, are embedded to design a user interface guideline for educational semantic application. 
The International Journal of Multimedia \& Its Applications (IJMA) Vol.10, No.6, December 2018

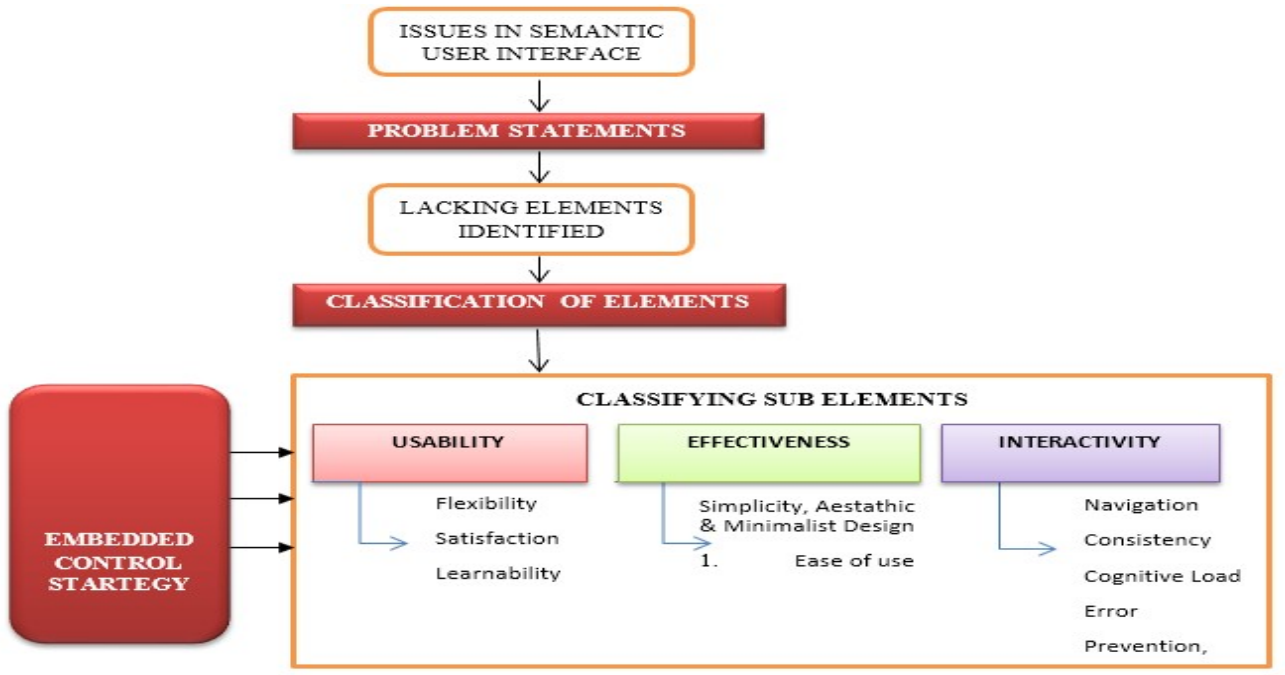

Figure 3. Task undertaken in producing the user interface guideline

This section discusses on the evaluation of user acceptance towards the developed prototype. In this phase participant are given chance to use and evaluate the prototype in order to validate if the research objectives whether they are obtained. These evaluations are carried out to answer these research questions:

- Does the developed user interface with proposed Semantic User Interface Guideline supports educational semantic web application?

- $\quad$ Does the developed user interface with proposed Semantic User Interface Guideline(SUIG) accepted by the user?

- Does the usability, effectiveness and interactivity occur in the design of user interface with the proposed Semantic User Interface Guideline (SUIG)?

\subsection{Prototype of user interface with proposed Semantic User Interface Guideline (SUIG). (Objective 2)}

An informative layout which consist the following semantic elements was explained. The element are simplicity (minimalist design), Ease of Use and Consistency.

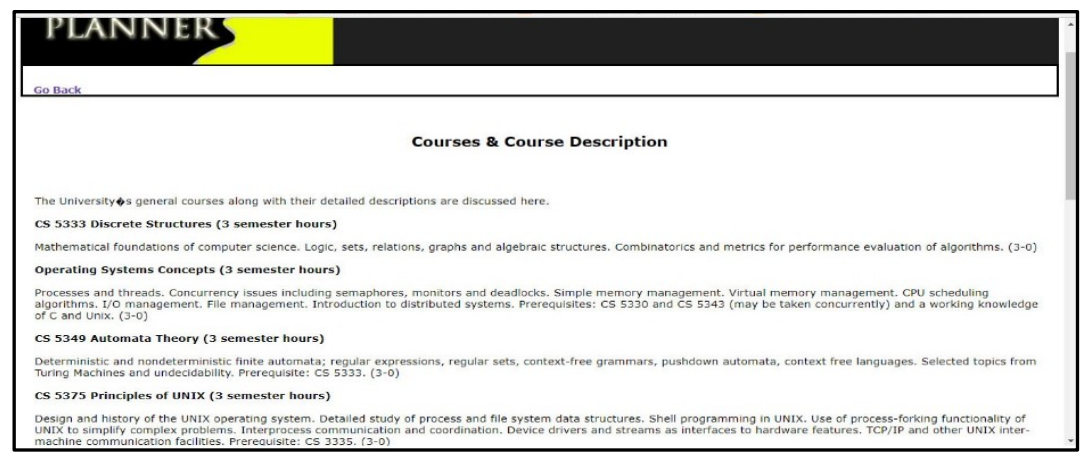

Figure 4.Descriptive page, where user would be acknowledged prior their interaction with the system's functionalities and contents. 
The International Journal of Multimedia \& Its Applications (IJMA) Vol.10, No.6, December 2018 When user could remember what path that they partake in order to achieve their target goal, a sense of urgency and satisfaction could be aroused from each of their participation with the system. Therefore, the system is designed together with the motivation of encouraging good rate of remembrance. If user could perform their task with fluid dexterity with each unique visits, then it is proven that their mind could remember and correlate with the on-screen elements well. In this section, an informative layout which consisting the following semantic elements was explained. The elements are learnability, flexibility, navigation, cognitive load and affordance.

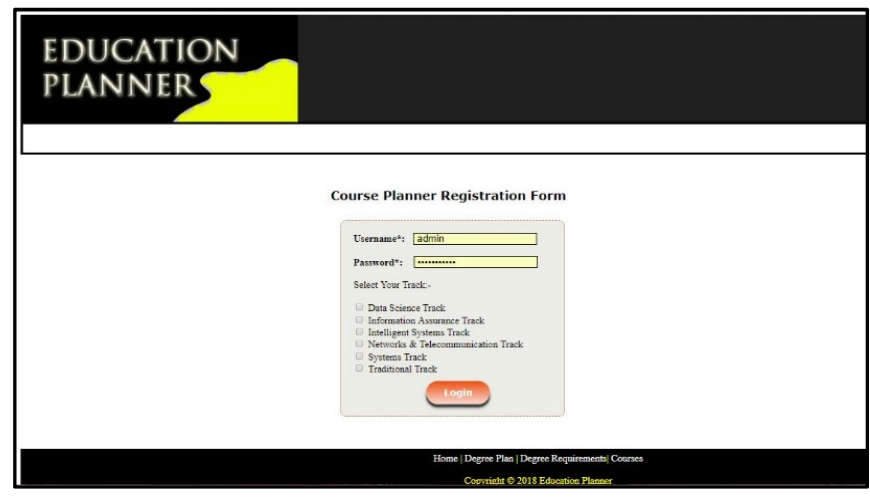

Figure 5.User registration page, where they need to add in their credentials before being allowed to involve in any of the system's functionalities

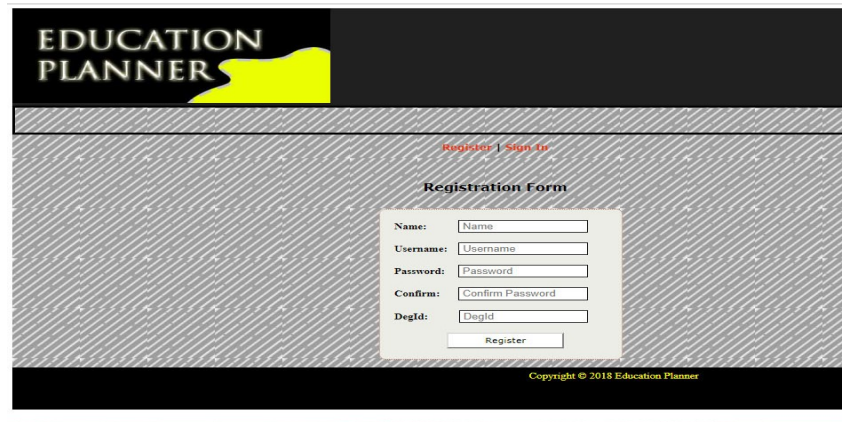

Figure 6.Registration page. Each function that includes user keying in their particulars is designed in a monotone, uniformed pattern so as to improve retention rate

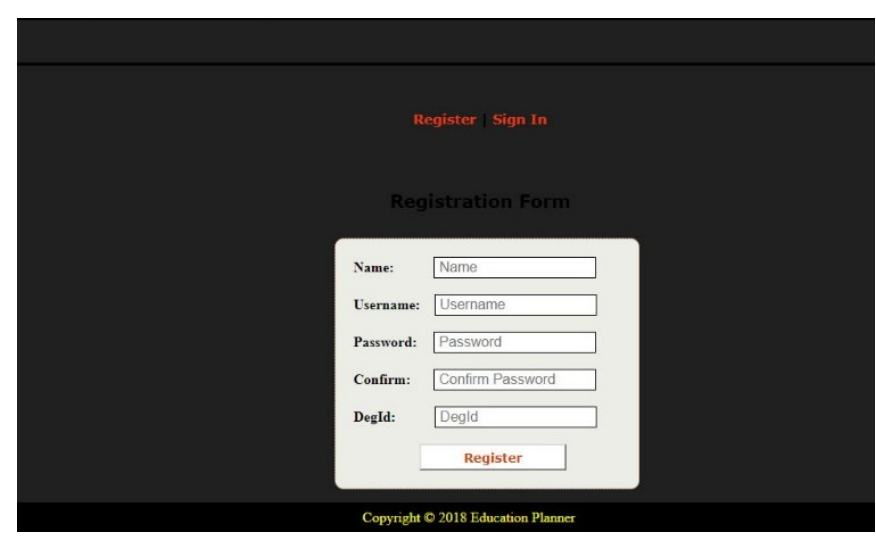

Figure 7.Admin registration page. 
The exclusivity theme exudes an importance feel for user who possesses more control over the system, in this case system administrators.

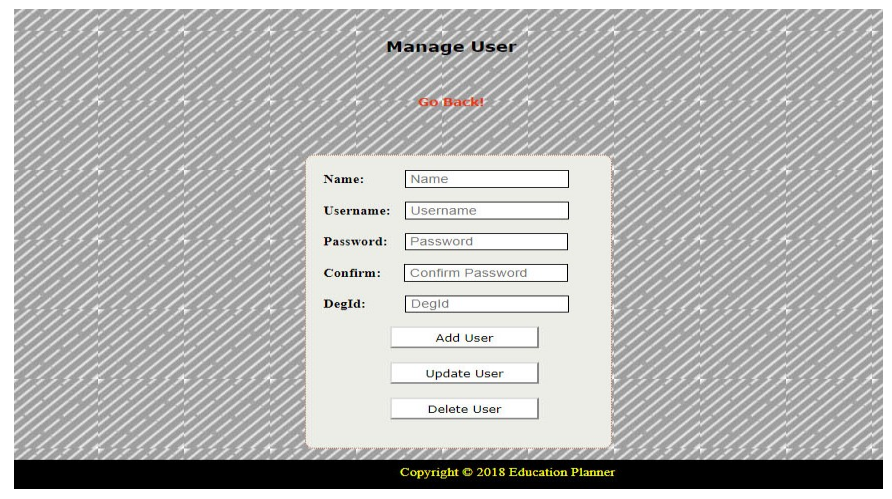

Figure 8. User management page

Although simple and does not contain many critical decision making aspects, the interface elements ensure that the system is easy to navigate and operate thus reducing trauma for first time user and encourage more frequent visits.

\subsection{Result of User ACCePTance Towards DeVeloped User Interface With Proposed Semantic User InTERface Guideline (SUIG). (ObJective 3)}

\section{VISIBILITY OF SYSTEM STATUS}

In component "visibility of system status user" was evaluated on whether the users are informed on the function of the application. User should always be informed by the application; they should also receive proper informative feedback from the application in appropriate time. It depicts the functions in the web application. For instance, when a user is in the front page of the application does the user know on what to do. Result of the interview shows that P2, P3, P4, P6, P7, P8 and P10 agrees that they are alert with the functions in IRPH which resulted seven out of ten participant chooses IRPH whereas P1, P2, P3, P4, P6, P7, P8 and P10 agrees that they are aware of the functions in SUIG which resulted eight out of ten participant chooses SUIG. The participants mentioned that:

"In the Log In page in SUIG, I knew as a new user I will need to register as a user whereas in IRPH I was wondering on how should I log in without any Id"

"I was not informed on what to be done in the log in page in IRPH"

"I was left uninformed on where to get my materials in IRPH"

"There were selections for me to choose my materials in SUIG"

For this study, it concludes that the majority participant concurs that SUIG is more prominent in terms of the visibility of system status.

\section{MATCH BETWEEN SYSTEM AND THE REAL WORLD}

It is not rationale to use a system oriented language as it may confuse the user. A system should always consider using natural and understandable language relating to its users as well and at the 
The International Journal of Multimedia \& Its Applications (IJMA) Vol.10, No.6, December 2018

same time real-world rules complies in and a norm order. In this component user evaluates on whether the application uses simple phrases that the users are familiar with. For instance, in the page where the users were to create a lesson plans, the labels on the menus and the text are used similar to manual lesson plan templates. Thus, it enables the users who are teachers to use it

without any hesitation. Based on the interview result, for IRPH seven out of ten, P1, P2, P3, P5, P6, P8, P10 agree that they are able to do the task given whereas for SUIG, all the participant agrees that the contents used are familiar. One of the participants who were struggling in finishing the task explained that the reason was because SUIG uses fully English language and some on the term are not familiar as most of the manual lesson plans are in Malay Language. For this study, it concludes that the majority participant accepts that SUIG is more prominent in terms of match between system and the real world.

\section{USER CONTROL AND FREEDOM}

Users might select some options accidently in an application. In conjunction with that applications should always provide an "emergency exit" either to leave or to redo. In this component user had been evaluated on the actions when they accidently makes mistakes. In this case, it involves whether if they were allowed to go back to the previous page if they wanted to or they have options to redo or reset button. Based on the interview result, for IRPH seven out of ten participant, P1, P3, P4, P5,P6, P8, P9 and P10 agree that they are able to refine or go back when there is an error whereas for SUIG, nine out of ten participants, P1, P3, P4, P5, P6, P7,P8,P9 and $\mathrm{P} 10$ agrees that they are free to reset or redo the page. Statement from a participant proved this finding by telling that:

"The application allows me go back to the previous page as the application has design with a few pages and I can always reset back my work. IRPH has very less function and I will need to delete all and start all over again"

"IRPH doesn't have edit button or back button for me to view the information that I keyed in previously"

"It is so convenient to use SUIG as I can always go back to my previous work to edit"

For this study, it concludes that the majority participant accepts that SUIG is more accepted in terms of user control and freedom.

\section{CONSISTENCY AND STANDARDS}

The contents of the system should be presented in the same manner to ease the user and less memory used. Consistency here is that the idea that all the layout of headings, menus, layouts as well as buttons should be placed in the same place for every page. Based on the interview result, for IRPH has half of the whole ten participant, P4, P5, P7, P9 and P10 agree that the details, the buttons and the labels are consistent but one of the participant complained that the user could not remember where is the buttons as it was not in the same place before.

Whereas for SUIG, all ten participant agrees that throughout the application it maintains its consistency in terms of text, important buttons and menu and the designs are all in same standards for every pages. From this result, it concludes that the majority participant accepts that SUIG is more consistent and standardized. 
The International Journal of Multimedia \& Its Applications (IJMA) Vol.10, No.6, December 2018

\section{ERror Prevention}

Applications should apply a considerate and careful design in order to avoid problems or error with will lead to anxiousness in users. Error prevention here simply means the alternative the application has if in case there is any error. For example, the application provide "clear" button to clear input error by the user. Based on the interview result, none of the participant find error prevention function in IRPH whereas for SUIG, all ten participants agrees that the application provide them the error prevention by having clear button on important text fields. Moreover SUIG has a role as user support whereby in that page users are allow to SMS or submit comment to the system administrator. A participant mentioned that:

"It is so convenient for me to clear certain text field especially the whole text field where I need to fill up long texts"

And another participant agrees that:

"I can actually send an SMS or comment in separate area"

"There is SMS button in the application which I can use if I encounter problem"

For this study, it concludes that the majority participant accepts that SUIG is more prominent in error prevention.

\section{RECOGNITION RATHER THAN RECALL}

Applications should make labels, text, selections, icon buttons and instructions clear and understandable so that participants are not required to remember all the unnecessary information. In this component, participant should be aspect that the instructions in the applications are clearly available. Especially those parts where the users need to key in or input certain information by themselves and those parts where there is no selection button; users should know what to key in. Based on the interview result, only three out of ten participants, P4, P9 and P10 agrees that they were clear in what to key-in in IRPH application.

One of the participant mentioned that "I will need to go to the manual button all the time which is in another page and get the manual in order to understand on what I should do".

Another participant claims that, "There are no instructions at all in IRPH to refer to".

Whereas, for SUIG, all ten participants agrees that the application provide each set of instruction for each and every functions and user are not left wondering. Here some statements from the participant who agrees with SUIG, "SUIG has instruction in all the interface in a consistent place", "I am well aware of function of each field". From this result, it concludes that the majority participant accepts that SUIG is clear and visible in terms of instructions.

\section{FLEXIBILITY AND EFFICIENCY OF USE}

Applications should always provide effective services for both experience and novice users with specified options. In this component, user as a novice user should aspect many alternative for certain function for example rather than typing all the information they should be able to choose from dropdown list, checkboxes or radio button. Based on the interview result, only two participants out of ten participant, P4 and P6 agrees that they were given alternative in performing 
The International Journal of Multimedia \& Its Applications (IJMA) Vol.10, No.6, December 2018

certain tasks in IRPH application whereas for SUIG, only four participant out of ten participant, P1, P3, P7, P8, and P10 agree that as a new user they were given other alternatives to finish the tasks. In this matter, both the application has constraints since both are not providing enough alternatives for new users. However, a participant does appreciate on the features on both IRPH and SUIG, as there were dropdown lists on subjects and checkbox options in SUIG that allows the participant to choose more than one options.

\section{Aesthetic ANd Minima List Design}

Application should not show excessive and unnecessary info and designs because that will make users confused on using the function in the application. The key is to produce an overall simple and minimalist design with applicable information without the inapplicable materials. Brief wordings and classification of subjects are associated in the design as well as the overall appearance on the application should be easily seen without any exaggerating designs. Based on the interview result, all ten participants agree that both IRPH and SUIG application has minimal design and simple information that ease the user to access. This is proven by participant when they mention that:

"in the application (SUIG) has less design and same colours in all the pages so I can somehow know there the buttons and menus are in each pages."

"IRPH uses light colours as background which eases me to see the whole system"

"SUIG uses white background which I think better for website which has a huge amount of text projected"

"IRPH has a similar and readable font which makes it easily read."

"SUIG has more functions than IRPH but it is not congested as lest design elements applied"

\section{HELP USERS RECOGNIZE, DIAGNOSE AND RECOVER FROM ERRORS.}

The error messages should not only project errors it needs to notify the problems and also propose solutions for users on how to prevent. In this component user should get feedback for errors they made and at the same time and suggest solutions for users. Based on the interview result, five participants out of ten participant, P2, P5, P7, P8 and P9 agree that IRPH has error projection especially in "search" button, when the user key in something irrelevant it displays " 0 result" whereas for SUIG application six participant out of ten participant, P1,P2, P4, P5, P8 and P9 agrees that they we informed about the errors and together a solution for the errors. For example, when a user key in the wrong keyword, a pop up message appear informing on the error and what user should key in instead. For this study, it can be concluded that the majority participant accepts that SUIG is more helpful in helping users to recognize, diagnose and recover from errors.

\section{HELP AND DOCUMENTATION}

It is important for applications to provide help and documents that should be easy to search and they make effort to support users with problems. This component allows user be alert with the errors they made. The users are informed if there is an error in task they perform. 
The International Journal of Multimedia \& Its Applications (IJMA) Vol.10, No.6, December 2018

Based on the interview result, all ten participants mention that IRPH does not project any error messages so they do not really know if they key in the right information. In contrast in SUIG application all ten participants agree that they are alerted by error messages when they input wrong information. This can be seen when one of the participant commented that the user were having trouble logging in as there an error message popped out telling that the participant keyed in the wrong password. From this result, it concludes that the majority participant accepts that SUIG is highly contented in terms of help and documentation.

\section{SUMMARY OF FINDINGS}

Based on the results of the comparative study eight out of ten participants agrees that they are more comfortable in using interface in SUIG compared to IRPH. Five out of ten participants accepted that the interface on IRPH is easy to use. Thus, bringing to a conclusion that SUIG is more accepted by user than IRPH.

Overall, this research was carried with three primary objectives. The first objective is to produce a user interface guideline for educational semantic application. The second objective is to develop a user interface design with the proposed user interface guideline. Lastly, the third objective is to evaluate user acceptance towards the user interface prototype which was developed based on the proposed user interface guideline to understand their benefits and drawbacks. In this chapter, the development of user interface guideline for educational semantic application will be discussed. The implication of evaluating user acceptance towards the developed prototype will also be elaborated. Finally, this study will provide the contribution of the research work as well as recommendation for future work.

\begin{tabular}{|c|c|c|c|c|c|c|c|c|c|c|}
\hline Heuristic Evaluation (IRPH) & P1 & P2 & P3 & P4 & P5 & P6 & P7 & P8 & P9 & $\mathbf{P 1 0}$ \\
\hline Visibility of System Status & $\mathbf{x}$ & f & / & / & $\mathbf{x}$ & f & / & $/$ & $\mathbf{x}$ & f \\
\hline Match between system and the real world & / & f & f & I & I & f & 7 & 7 & I & f \\
\hline User control and freedom & / & $\mathbf{x}$ & f & $\mathbf{x}$ & / & / & $\mathbf{x}$ & $/$ & f & / \\
\hline Consistency and Standards & $\mathbf{x}$ & $\mathbf{x}$ & $\mathbf{x}$ & / & f & $\mathbf{x}$ & f & $\mathbf{x}$ & / & f \\
\hline Error prevention & $\mathbf{x}$ & $\mathbf{x}$ & $\mathbf{X}$ & $\mathbf{x}$ & $\mathbf{x}$ & $\mathbf{x}$ & $\mathbf{x}$ & $\mathbf{x}$ & $\mathbf{X}$ & $\mathbf{x}$ \\
\hline Recognition rather than recall & $\mathbf{X}$ & $\mathbf{X}$ & $\mathbf{x}$ & / & $\mathbf{x}$ & $\mathbf{x}$ & $\mathbf{x}$ & $\mathbf{x}$ & $f$ & f \\
\hline Flexibility and efficiency of use & $\mathbf{x}$ & $\mathbf{x}$ & $\mathbf{x}$ & f & $\mathbf{x}$ & f & $\mathbf{x}$ & $\mathbf{x}$ & $\mathbf{x}$ & $\mathbf{x}$ \\
\hline Aesthetic and minimalist design & $f$ & f & f & / & $/$ & f & f & / & $/$ & $f$ \\
\hline $\begin{array}{l}\text { Help users recognize, diagnose and recover } \\
\text { from errors }\end{array}$ & $\mathbf{x}$ & / & $\mathbf{x}$ & $\mathbf{x}$ & / & $\mathbf{x}$ & / & / & / & $\mathbf{x}$ \\
\hline Help and documentation & $\mathbf{X}$ & $\mathbf{x}$ & $\mathbf{X}$ & $\mathbf{X}$ & $\mathbf{x}$ & $\mathbf{x}$ & $\mathbf{x}$ & $\mathbf{X}$ & $\mathbf{X}$ & $\mathbf{X}$ \\
\hline Heuristic Evaluation(SUIG) & P1 & P2 & P3 & P4 & P5 & P6 & P7 & P8 & P9 & $\mathbf{P 1 0}$ \\
\hline Visibility of System Status & I & 1 & 1 & I & $\mathbf{x}$ & 7 & I & I & $\mathbf{X}$ & 7 \\
\hline Match between system and the real world & f & / & / & $\mathbf{x}$ & / & / & $\mathbf{x}$ & / & $\mathbf{X}$ & $/$ \\
\hline User control and freedom & f & $\mathbf{x}$ & / & / & / & / & / & / & $/$ & I \\
\hline Consistency and Standards & f & f & f & f & f & f & f & / & f & f \\
\hline Error prevention & f & f & / & / & / & / & / & / & / & f \\
\hline Recognition rather than recall & / & / & / & / & $/$ & / & / & $/$ & / & / \\
\hline Flexibility and efficiency of use & f & f & / & $\mathbf{x}$ & $\mathbf{x}$ & $\mathbf{x}$ & f & / & $\mathbf{x}$ & f \\
\hline Aesthetic and minimalist design & f & / & / & / & / & f & $/$ & / & / & $/$ \\
\hline $\begin{array}{l}\text { Help users recognize, diagnose and recover } \\
\text { from errors }\end{array}$ & / & f & $\mathbf{x}$ & / & / & $\mathbf{x}$ & $\mathbf{x}$ & / & / & $\mathbf{x}$ \\
\hline Help and documentation & f & f & f & f & f & / & / & / & f & $/$ \\
\hline
\end{tabular}

\section{The DeVelopment of USER InTERface Guideline for Educational Semantic APPLICATION}

The main target of this research is to produce a user interface for the educational semantic application in order to facilitate and promote semantic content authoring among Web end-users. Needs assessment is the method to problem identification and their causes, then rectifying the solutions to those problems [19]. 
The International Journal of Multimedia \& Its Applications (IJMA) Vol.10, No.6, December 2018

To achieve this goal, researcher gathered information from the related journal from Web of Science, IEEE and Science Direct on the guidelines. This group of journals were chosen based on the most cited journal articles as the quality and standards of journals complies to the impact factor statistics. Thus, those journal with most cited counts tend to be more reliable and legit in the group of top tier journals [24]. An analysis process was done using three guidelines to identify possible components for the proposed user interface guideline. Another process of analysis was done from the same group of journals to identify the constraints of existing semantic application. The third analysis process was to embed in Cognitive Load Theory which involves three different types of cognitive load which is intrinsic cognitive load (ICL), extraneous cognitive load (ECL) and Germane cognitive load (GCL). A control strategy was designed out of these three cognitive loads to control the total cognitive load of the user interface guideline for educational semantic application [25].

Gathering information from all three analysis processes contributed in a user interface guideline for educational semantic web. The user interface is then validated by the experts and the comments received are treated as a refinement.

\section{The Evaluation of User ACCePtance towards the Developed Prototype}

An evaluation has been conducted on the existing educational semantic application and on the prototype developed based on the proposed semantic user interface guideline. The evaluation measures the user acceptance towards both educational semantic applications. Based on the findings of the research, it is proven that the user interface prototyped with the proposed user interface guideline was more accepted as compared to the application that does not have any guideline. Eight of the ten overall participants agree to use the prototype user interface with proposed guideline. This findings is in line with the findings of [26] indicates that focusing and anticipating is exactly what user should be concerned about and ensuring that the interface has components that's enables users to easily accessible, understandable, and use to facilitate in accomplishing those actions. The user interface design is a combination of concepts between interaction design, visual design, and information architecture. The components used in the development of the prototype can significantly cater to the needs of the teachers as well as the novice users. A few refinements have to be made in terms of feedback given by the users before it is decided to be commercialized and also taking into consideration the stages of a typical user interaction with a generic interaction system [5]. From the findings, it also realized that the amount of time, constraint and effort that a participant is undertaking depends on the usability, effectiveness and interactivity of a user interface which give a big impact in cognitive load. By decreasing the Intrinsic Cognitive Load (applying clear and precise instruction, two types of typography as well as availability in sequence process), decreasing Extraneous Cognitive Load (avoiding redundant information and link, avoiding long messages) and increasing Germane Cognitive Load (clear explanation and present material in a way that capture attention has contributed to effective learning process. This statement is synchronised with the solution for the statement of [27].

\section{The Advantages and Disadvantages of The Developed Prototype}

The advantages of the developed prototype is that the participants were constantly informed about each and every task by the prototype, thereby costing them less time to complete the task. Participants are given the choice to edit or redo the inputs that they keyed in whenever they made mistakes. Throughout the activity, the prototype maintained its consistency in terms of all the 
The International Journal of Multimedia \& Its Applications (IJMA) Vol.10, No.6, December 2018 important buttons, menu, the design and the alignment to be same in all the pages. The prototype consistently provided error prompt together by having clean button and important text field. This is helpful because it directs the participant straight to the task rather than lingering around the application.

Aside from that, all instructions were given throughout the application. Instruction was given in each input field to help the participant on what and where to go, in order to complete a certain task. Hence, the edge of the application include less design and use of selective colour in the overall application, which will eventually use up less cognitive load and less confusion on the part of the participants. Obtaining error prompt, together with its solution immediately, in case the participant made any mistakes, resulted to the convenient use of the prototype.

However, the prototype has several constraints that also needs to be looked upon. One of the issues is the unfamiliarity of the participants on some terms and functions. This is due to the fact that the whole applications are in English and some are in highly technical terms. It is recommended therefore, that for the prototype to have a dual language system to ease the users. Another constraint is that then participants were required to type in or input the material and instructions. One suggestion for this constraint is to have more dropdown lists to ease the inconvenience experienced by users.

\section{CONCLUSION}

Various constraints have been highlighted regarding semantic application. Due to its nature, a lot of on-going researches seemed to be focused more on the back-end aspect which covers the linked data, knowledge representation and semantic metadata being produced, and there is less attention is given to the front line of the semantic application. This research has addressed the aforementioned gap or constraint in semantic application through the creation of Semantic User Interface Guideline (SUIG) for educational semantic application. This guideline could be used as future guideline to upcoming educational semantic application and this method will somehow get to encourage the web manager to pay more attention to the front line as this is the medium where the users interact. Another major research contribution is the utilisation of the existing user interface guideline in the semantic web application user interface.

Overall, this research proves that the proposed user interface guideline for educational semantic application does significantly fulfil all the aspects of usability, effectiveness and interactivity. The Jakob Nielsen's Heuristic Evaluation has assisted the researcher to examine the implication of the developed prototyped based on proposed Semantic User Interface Guideline towards the user's acceptance. This report is made to ease the users to retrieve information from the educational semantic application as well as to be a guideline to future semantic application. Through this study, it has proven the importance of user interface in semantic application by being an eye opener, especially on the part of the upcoming web designers. This research could be definitely further be established by considering inputs given and retrieved throughout the research process.

\section{REFERENCES}

[1] Maedche, A., Staab, S.(2001), "Ontology learning for the semantic web", IEEE Intelligent Systems, 16(2).

[2] Aslina,S., Chung, P. W. H., Dawson,C. W.(2011). "Case Representation and adaptation in SmartLP A Web-based Lesson Planning System”. ICAART (1) 2011: 440-445. 
The International Journal of Multimedia \& Its Applications (IJMA) Vol.10, No.6, December 2018

[3] Myers,B.A., Rosson, M. B.(1992). "Survey on user interface programming". ACM press, New York, NY, USA, Pages 195-202.

[4] Czerkawski, B., \& Hernandez,J. (2012) "Formal, non-formal, informal E- Learning experiences with emerging technologies: A case study of a graduate educational technology program".(pp. 337-355). , IGI Global.

[5] Sun, Q., Abdourazakou Y., \& Norman, T. J. (2017) "Learn Smart, adaptive teaching, and student learning: An empirical investigation", Journal of Education for Business, (pp: 36-43).

[6] H. L. Woo (2009), "Designing multimedia learning environments using animated pedagogical agents: factors and issues", Computer Assisted Learning, no. 25, pp. 203-218, 2009.

[7] Mitcham, C. (1994). "Thinking through technology: the path between engineering and philosophy". Chicago: The University of Chicago Press. OMG-UML (2003). UML Specification, version 1.4. Retrieved October 14, 2003

[8] Abdelmalak, M.M.M.(2015), "Web 2.0 technologies and building online learning communities: students' perspectives". New Valley College of Education, Assiut University, Egypt

[9] Nanavati,A. (2005)."User interfaces for semantic web applications. Knowledge management systems". School of information, The University of Texas at Austin

[10] Koper, R. (2004). "Use of the semantic web to solve some basic problems in education", Journal Of Interactive Media in Education.

[11] Staab, S., Maedche, A., Handschu, S. (2001)." An annotation framework for the semantic web". In S. Ishizaki, Proc. of The First International Workshop on Multimedia Annotation, Tokyo,Japan

[12] Harvey, Craig \& J. Koubek, R \& Rothrock, Ling \& Darisipudi, A \& Kim, Jong W \& Munch, J. (2005). "Cognitive Ergonomics." (pp:70-89).

[13] Aslina, S. (2016). "Knowledge representation of lesson plan domain for CBR application". International Conference on ICT in Education 2016, Universiti Pendidikan Sultan Idris.

[14] Jason, O. (2008). “The Semantic Web in Education”.EDUCAUSE Quarterly, vol. 31, no. 4

[15] Siipo, A. Huttunen S., Jukka, H., Väyrynen, E., Keskinarkaus, A., László, M., Juuso, I.,Laukka, S.,\& Seppänen, T.,"A Multimodal Observation and Analysis System for SocialInteractions".Forthcoming in2014. MORE

[16] Breslin, J., Passant, A., and Decker, S. (2009). The Social Semantic Web. SpringerVerlag,Heidelberg

[17] Alexander M., Steffen S. (2001). "Learning and the semantic web". Institute AIFB, University ofKarlsruhe.

[18] Roblyer M. D. (2015)." Introduction to systematic instructional design for traditional, online, and blended environments". University of Minnesota, Nova Southeastern University

[19] Dick, Carey (2009)," The Systematic design of instruction", 7th edition, Pearson Publishing

[20] Damljanovic, D., Agatonovic, M., Cunningham, H. (2010). "Natural language interfaces to ontologies: combining syntactic analysis and ontology-based lookup through the user interaction". Semantic Web: Research and Applications.

[21] Auer, S.,Demter. J., Martin. M.,Lehmann. J.. (2012). “An extensible framework for high performance dataset analytics". 18th International Conference, EKAW 2012, Galway City, Ireland. Proceedings, volume 7603 of Lecture Notes in Computer Science, (pp 353-362), Berlin.

[22] Luciano S., Sofia C., Massimo T., Marco B., Silvia L., Marco R., Elena C., Ivan D (2015). "ePlanning: Ontology-based system for building individualized education plans for students with special educational needs". Media Education, volume 6.

[23] Hsu \& Leslie (2017). "Visualization and interaction for ontologies and linked data \&semantic statistics"., Journal Of Web Semantic.

[24] Haghdoost, A., Zare, M., \& Bazrafshan, A. (2014). "How variable are the journal impact measures? Online Information Review, 38(6), 723-737,

[25] Sweller, J. (2010). "Element interactivity and intrinsic, extraneous and germane cognitive load". Educational Psychology Review, 22, 123-138.

[26] Mendoza, Gonzalez, Ricardo (2016). "User-Centered design strategies for massive open online courses (MOOCs)". 
The International Journal of Multimedia \& Its Applications (IJMA) Vol.10, No.6, December 2018

[27] Ton, J. (2010). "Cognitive load theory, educational research, and instructional design: some food forthought Instructional Science", Volume:38, (pp:105-134).

[28] Mehrdad, H. (2007). "Art of teaching", Tehran: Ravan Publication.

[29] Lassila, O. (2007). "Programming semantic web applications: a synthesis of knowledge representation and semi-structured data", Ph.D. dissertation, Helsinki University of Technology

\section{Authors}

Tinashini Jayadevan Naidu is a secondary school teacher in an international school (Wesley Methodist International School Kuala Lumpur). She is currently doing her Master's in Education (Multimedia) in Universiti Perdidikan Sultan Idris. Her research interests are on Multimedia in Education and Human-Computer Interaction. She is teaching ICT for Year 8, 9 \&ICT IGCSE, Cambridge Syllabus for Year 10\&11.

Dr. Aslina Saad is an Assoc. Prof. in Computing Department, Faculty of Art, Computing and Creative Industry UPSI. She obtained her $\mathrm{PhD}$ in Computer Science from University of Loughborough, England in 2011. She is a graduate in Master of Science (Information Technology), UTM and Bachelor of Information Technology (Science and System Management), UKM. Her research interests are on Computer in Education, Information System, Artificial Intelligence and Software Engineering. She has been teaching since 2002 in various courses such as Database System, ICT principles, System Analysis \& Design, Software Design, Software Project, Software Process, Information System Development and Object Oriented Analysis \& Design.

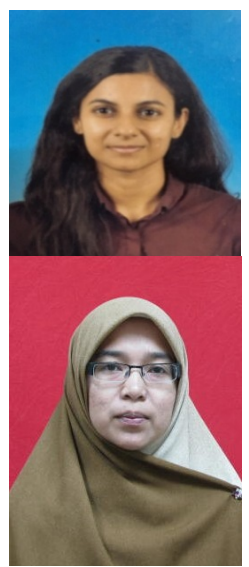

